\title{
Applications of agent-based simulation for human socio-cultural behavior modeling
}

\author{
Hong Jiang, Waldemar Karwowski, and Tareq Z. Ahram ${ }^{1}$ \\ Institute for Advance System Engineering, Department of Industrial Engineering and Management Systems, Uni- \\ versity of Central Florida, Orlando, FL 32816, USA
}

\begin{abstract}
Agent-based modeling and simulation (ABMS) has gained wide attention over the past few years. ABMS is a powerful simulation modeling technique that has a number of applications, including applications to real-world business problems [1]. This modeling technique has been used by scientists to analyze complex system-level behavior by simulating the system from the bottom up. The major application of ABMS includes social, political, biology, and economic sciences. This paper provides an overview of ABMS applications with the emphasis on modeling human socio-cultural behavior (HSCB).
\end{abstract}

Keywords: Agent-based modeling, Human Socio-cultural Behavior, simulation.

\section{Introduction}

Agent-based modeling and simulation (ABMS) is also called Agent-based modeling (ABM) or Agentbased simulation (ABS). ABMS has gained increasing attention over the past 10 years [2]. Many research papers focus on ABMS applications in the areas of crowd flow simulation, and organizational behavior. The behaviors in an ABMS support an agent-level to system-level behaviors. Scientists use ABMS to analyze complex systems by simulating systems from the bottom up. The goal of this paper is to provide an overview of ABMS applications with the emphasis on modeling human socio-cultural behavior (HSCB).

\section{ABMS and its applications}

\subsection{ABMS Concept and its Benefits}

ABMS is a computer technique to simulate complex systems whose components are agents. Generally speaking, an ABMS is a hybrid discrete- continuous simulation model with proactive, autonomous, and intelligent entities. The pro-activeness refers to the ability of taking initiative. Agents can initiate actions, communicate with other agents, and make decisions on their own [3]. With the increasingly complex world we live in, there exists a need to analyze and understand the world and system better than before. This means that the ABMS paradigm can represent large systems consisting of many subsystem interactions. Although computer simulation as an analytical tool has been around since the advent of computers, the ability of the ABMS paradigm to simulate the complex systems has moved it into a breadth of fields ranging from engineering to mathematics to social science and to economics [4]. By sufficiently understanding ABMS, scientists would have a better understanding of the dynamics of the process and how systems components behavior changes over time. During the recent years, researchers combine ABMS with neural networks, evolutionary algorithms and other learning techniques to provide better solutions for complex systems.

\footnotetext{
${ }^{1}$ Hong Jiang, Waldemar Karwowski, and Tareq Ahram

Institute for Advance System Engineering, Department of Industrial Engineering and Management Systems, University of Central Florida,jianghong@knights.ucf.edu,wkar@mail.ucf.edu,tareqahram@gmail.com, Orlando, FL 32816, USA
} 


\subsection{Understand an Agent}

There is no universal agreement on the precise definition of the term "agent" in the context of ABMS. According to Macal and North [2], agents have certain characteristics. An agent has its own attributes, rules of behaviors, memory, and decision making ability and is able to adapt its behavior into environment. Figure 1 shows the agent characteristics by UML.

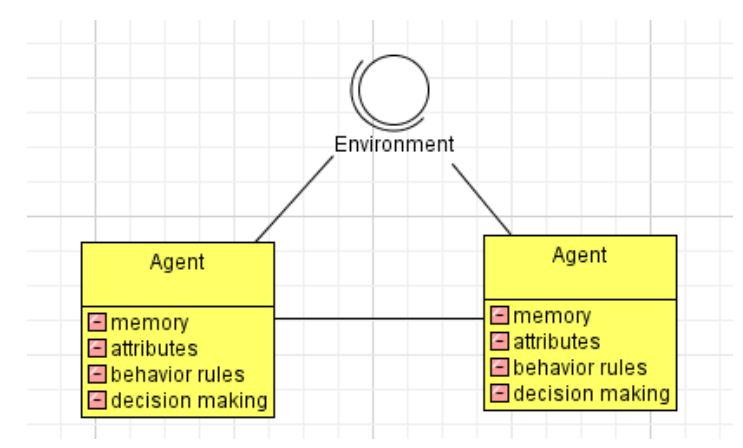

Fig 1: Agent characteristics (UML interface)

An agent also has the following characteristics[2]:

- An agent is identifiable.

- An agent is situated, living in an environment with which it interacts along with other agents.

- An agent may be goal-directed, having goals to achieve with respect to its behaviors.

- An agent is autonomous and self-directed.

- An agent is flexible, having an ability to learn and adapt its behaviors based on experience.

The fundamental feature of an agent is the capability of the component to make independent decisions. This requires agents to be active rather than purely passive.

\subsection{ABMS Applications}

Currently, ABMS has been used in different field of study such as economics, social science, military, biology and public policy. In the paper of Heath et al. [4] they show ABMS being used by fields whose systems involve many interacting autonomous entities. This supports the fundamental belief that ABMS is good at modeling and analyzing interacting entities.
Barabasi [5] listed examples of emergent phenomena in the social, political and economic sciences. The situation of interest where emergent phenomena is classified into four areas:

1. Flows: evacuation, traffic, and customer flow management.

2. Markets: stock market and software agents, and strategic simulation.

3. Organizations: operational risk and organizational design.

4. Diffusion: social context, business applications.

During the recent years, scientists have used ABMS to simulate Human behaviors because the ability of the ABMS paradigm to simulate the complex systems.

\subsection{Top used Software for ABMS}

Among others, the following are the most commonly used software platforms for ABMS: Anylogic, MATLAB, Swarm, Repast and NetLogo. Among these software, only Anylogic and Matlab are commercial packages.

Swarm was the first ABMS software development environment launched in 1994 at the Santa Fe institute. Following the original Swarm innovation, Repast (Recursive Porous Agent Simulation Toolkit) illustrates the state-of-the-art in agent-based modeling toolkit [6]. Repast has been used extensively in social simulation applications.

\subsubsection{NetLogo ${ }^{\circledR}$ for Simulating ABMS}

NetLogo ${ }^{B}$ [7] is a good example of the ABMS tool. NetLogo ${ }^{B}$ is a multi-agent programming and modeling environment based on JAVA platform. It is a powerful tool in many fields of research and education. It is especially well suited for modeling large collections of interacting of independent agents developing over time, so it is a promising solution for simulating and analyzing complex problems. NetLogo $^{\circledR}$ is convenient platform for the analysis of any complex system developing over time as the programmer can give instructions to thousands of agents all operating concurrently [8]. It is widely used by new programmers who do not have a lot of experience in creating complex programs. 
Figure 2 shows an example of NetLogo ${ }^{\circledR}$ modeling different personalities between Altruism and Selfishness.

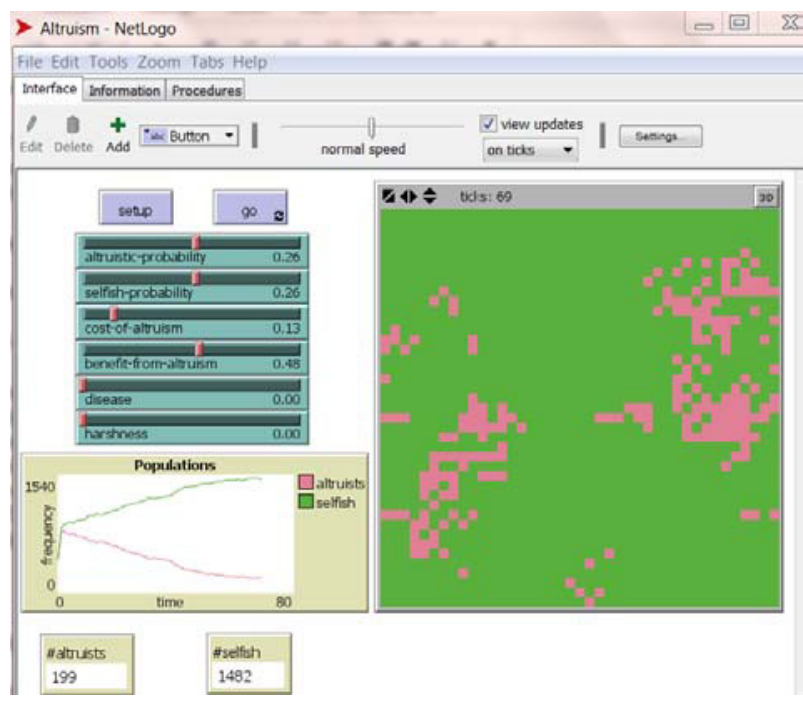

Fig 2: Screenshot of simulating Social Science using NetLogo ${ }^{\circledR}$

NetLogo ${ }^{\circledR}$ also has a plenty of sources from its library including of Art, Biology, Chemistry \& Physics, Computer Science, Earth Science, Games, Mathematics, Network, Social Science and System Dynamics. This function made it very useful for different fields of studies. Researchers can use NetLogo to do programming according to their different goals.

\section{ABMS and HSCB}

\subsection{ABMS in Social Behavior Research}

Social behavior research is one component of the HSCB. Scientists cannot get a better understanding of HSCB without a good basis in social behavior. Social science is not only primarily concerned with the behavior of isolated individuals but also concerned with the behavior of interactions among individuals [9]. Social scientists have been exploring the factors that contribute to the human social behavior over a period of time and where it has lead them in this field for employing ABMS to investigate this social phenomena. Social scientists found that ABMS is well suited for the social behavior because the systems is composed of interacting agents and exhibits emergent properties. In order to understand a politi- cal or economic system, scientists need to better understand how the individuals interact with each other, and how the results can be more than the sum of the parts. Human social interactions are complex which should consider social and psychological interactions.

In applications of ABMS to social behavior, agents represent people or groups of people, and agent relationships represent processes of social interaction [10]. Since agents can perceive and respond according to their environment and take the initiative to achieve their goals, ABMS was used by scientists to simulate their social behavior. For example, Marsell et al. [11] introduced PsychSim which allows scientists to explore multiple tactics for dealing with a social issue and potential consequences.

Schelling [12] is credited with developing the first social agent-based simulation in which agents represent people and agent interactions represent a socially relevant process back to 1978 .

Sociologists are using agent-based modeling to modeling social life as interactions among adaptive agents who influence one another in response to the influences they receive. Cognitive scientists are developing agent-based models of emotion, cognition, and social behavior based on the notion that a person's emotional state impacts their behaviors as well as their social interactions. Computational social science is becoming a subfield in the social sciences area [13].

In general, ABMS can be applied to social processing concepts and tools from social science and computer science. It represents a methodological approach that could ultimately permit two important developments: (1) testing or refining of existing theories that have proved to be difficult to formulate and evaluate using standard statistical and mathematical tools; and (2) a deeper understanding of the fundamental causal mechanisms in multi-agent. This leads to an overall better understanding and foundation in social behavior for HSCB.

\section{2. $A B M S$ and $H S C B$}

In 2008 the U.S. Department of Defense (DoD) established a new Research and Development (R\&D) program to develop a science base and associated technologies for modeling human, social and cultural behavior. The goal of HSCB modeling Program is to enable DoD and the US Government to understand and effectively operate in the human terrain during 
non-conventional warfare and other missions [14]. Since then, many scientists pay more attention to the HSCB and try to develop a methodology to better access and predict variability by applying different techniques to aid in studying the HSCB in military operations.

Modeling population-centric activities requires serious use of social science [15]. HSCB models are designed to help understand the structure, interconnections, dependencies, behavior, and trends associated with any collection of individuals. The HSCB modeling effort seeks to create models for social behavior from the small unit level, such as tribes, militias, small military units, terrorist cells, etc., to the macro level of nations, religions, cultures, ethnic groups and international organizations [16].

ABMS can be used to model HSCB and its benefits and applications are becoming very important to HSCB research area. By constructing such a model, social scientists aim to develop conclusions that provide insight into the human behavior of real world entities or phenomena. In addition, the models also provide a predicted behavior under certain circumstance [17]. For example, studies of insurgency warfare in U.S. military application, particularly the Army, seek to develop a methodology to better access and predict behaviors in human social cultural.

Past events that happened in Somalia, Iraq, and Afghanistan have made the military re-assess the $21^{\text {st }}$ century insurgency and revisit its strategy, operational concepts, organization and doctrine. Cultural, social and religious norms are strong drivers of behavior and they tend to be more persistent [18]. The U.S. military now focuses on understanding what a cultural, ethnic or religious group values and how to deal with property and leadership, with crime and redress, and which will provide a necessary foundation for working effectively with the population and their leadership.

However, there are still a lot of unknowns about the HSCB and the limitation of a model which will probably never be good at making exact predictions of what will happen. They can, however, be expected to provide information on the probabilities of various things happening. How to make an accurate model to simulate HSCB is very critical for scientists, which is also their goals to be achieved in the current society.

\subsection{Big Five Personality traits in $H S C B$}

The Five personality models were introduced by personality psychologists since early in the 1960s. Psychologists agree that personalities can cause different behavior and response. The "Big Five personality" traits include Neuroticism, Extraversion, Openness, Agreeableness, and Conscientiousness [19]. McCrace \& Costa [20] used different instruments to measure different personality and obtained results that correlated highly with the five trait scores of the Neuroticism, Extraversion, Openness Personality Inventory (NEO-PI) based on a six-year longitudinal study of trait-stability. This was later revised as the NEO Personality Inventory (NEO PI-R). Researchers have started to study personality traits in Human Social Cultural Behavior models during the recent years. For example, West [21] used the Big Five personality traits in Military applications.

Personality traits are related with job performance and training performance. Linking the Big Five personality Model with HSCB allows us to generate more accurate models for understanding human behaviors better.

\subsection{ABMS issues in HSCB}

ABMS has disadvantages like any other simulation techniques. The major current issue using ABMS to simulate HSCB is complex psychology and subjective choices making it difficult to quantify, calibrate, and calculate. HSCB researchers must be very careful of making decision on the basis of the quantitative outcome of a simulation that should be unbiased interpreted at the qualitative level. As mentioned before, ABMS can be developed and improve over time, but the complexities of social behavior and the current state of the related modeling art suggest that it is a really difficult process and it will take years for scientists to develop the whole methodology well. The challenge with HSCB research is to bring existing domains together, such as social behavior; cross-cultural behavior; personality traits etc., rather than create a new domain from scratch.

\section{Conclusion}

The new domain of HSCB modeling is gaining more and more interest within the M\&S community. 
Various approaches were used to simulate in HSCB and ABMS is one of these simulation tools. Although Agent-based Modeling has been developed for more than 10 years, its study in the field of the HSCB is very limited. There is not a single accurate model to combine the HSCB and ABMS together. Therefore, how to use ABMS to understand Human Social Cultural Behavior is a topic being discussed by scientists and researchers, ABMS has shown its advantages in this field. However, research indicates that HSCB modeling is a difficult process, ABMS is currently increasingly being used in the HSCB models.

\section{Future works}

Future work will concentrate on using ABMS such as NetLogo ${ }^{\circledR}$ to provide a platform and design a system

\section{References}

[1] E. Bonabeau, Agent-based modeling: Methods and techniques for simulating human systems. Colloquium Paper: Adaptive Agents, Intelligence, and Emergent Human Organization: Capturing Complexity through Agent-Based Modeling, Proceedings of the 2002 .

[2] C.M. Macal, M.J. North, Agent-based modeling and simulation. Winter Simulation Conference (WSC), Proceedings of the 2009, vol., no., pp.86-98, 13-16 Dec. 2009.

[3] W.K.V. Chan, Y.J. Son, Macal, Agent-based simulation tutorial - simulation of emergent behavior and differences between agent-based simulation and discrete-event simulation. Winter Simulation Conference (WSC), Proceedings of the 2010, vol., no., pp.135-150, 5-8 Dec. 2010.

[4] B. Heath, R. Hill, and F. Ciarallo, A survey of Agent-Based Modeling Practices. Journal of Artificial Societies and Social Simulation 12 (4)9.2009.

[5] A.L.Barabasi, Linked: the new science of networks, Cambridge, MA: Perseus Pub. 2002.

[6] M.L. North, N.T. Collier, J.T. Vos, Experiences in Creating Three Implementations of the Repast Agent Modeling Toolkit, ACM Transactions on Modeling and Computer Simulation, 16(1): 1-25 January 2006.

[7] U. Wilensky, NetLogo. Evanston, IL: Center for Connected Learning and Computer-Based Modeling, Northwestern University. 2009. Available via < http://ccl.northwestn.edu/netlogo/> access April 1, 2011].

[8] J. Al-Sharawneh, M.-A. Williams, ABMS: Agent-Based Modeling and Simulation in Web Service Se-lection. Management and Service Science, 2009. MASS '09. International Conference on, vol., no., pp.1-6, 20-22 Sept. 2009.

[9] R. Axelrod, L. Tesfatsion, A guide for newcomers to agentbased modeling in the social sciences.2005. Available via $<$ http://www2.econ.iastate.edu/tesfatsi/abmread.htm > [accessed March 27, 2011]. to understand the human social culture behavior, especially for understanding the impact of different personalities.

Design system dynamic models by using different simulation software to understand HSCB effects especially in the irregular situation such as in Military applications.

Use different scientific knowledge to increase understanding in the insurgency warfare. This may include scenarios of enhancing existing military capabilities.

[10] N. Gilbert, and K.G. Troitzsch, Simulation for the Social Scientist, UK: Open University Press.1999.

[11] S.C. Marsella, D.V. Pynadath, \& S.J. Read, PsychSim: Agent-based modeling of social interactions and influence. Proceedings of the international conference on cognitive modeling (pp. 243-248).2004.

[12] T.C. Schelling, Micromotives and macrobehavior. New York: Norton.1978.

[13] D. Sallach, C. Macal, The simulation of social agents: an introduction. Social Science Computer Review 19(3): 245248.2001.

[14] HSCB Modeling Program, 2009. Available viahttp://www.dtic.mil/biosys/docs/HSCB-news-spring2009.pdf

[15] A. Tolk, P.K. Davis, W. Huiskamp, G.L. Klein, H. Schaub, \& J.A. Wall, Towards Methodological Approaches to Meet the Challenges of Human, Social, Cultural, and Behavioral (HSCB) Modeling.2010.

[16] The Defense Technical Information Center (DTIC®). 2011. Available

via http://www.dtic.mil/dtic/stresources/dticreview/hscNbm/inde x.html

[17] X.H. Cui, T.E. Potok, A Particle Swarm Social Model for Multi-Agent Based Insurgency Warfare Simulation. Software Engineering Research, Management \& Applications. SERA 2007. 5th ACIS International Conference on, vol., no., pp.177-183, 20-22 Aug. 2007.

[18] S.K. Numrich, Introduction to Architectures: HSCB Information-What It Is and How It Fits (or Doesn't Fit).2010.

[19] L.R. Goldberg, Language and individual differences: The search for universals in personality lexicons. Review of personality and social psychology, 2, 141-165. 1981.

[20] R.P. McCrae, \& P.T. Costa, The structure of interpersonal traits: Wiggins's circumplex and the five-factor model. Journal of personality and social psychology, 56(4), 586. 1989.

[21] P.D. West, \& F. McNair, Human, Social, and Cultural Behavior Modeling for Stability, Security, Transition, and Reconstruction Operations. Center for Technology and National Security Policy, 28, 30. 2008. 